\section{Endoscopic ultrasound-guided thrombin injection in a large splenic artery aneurysm: first report in a patient with tropical chronic pancreatitis}

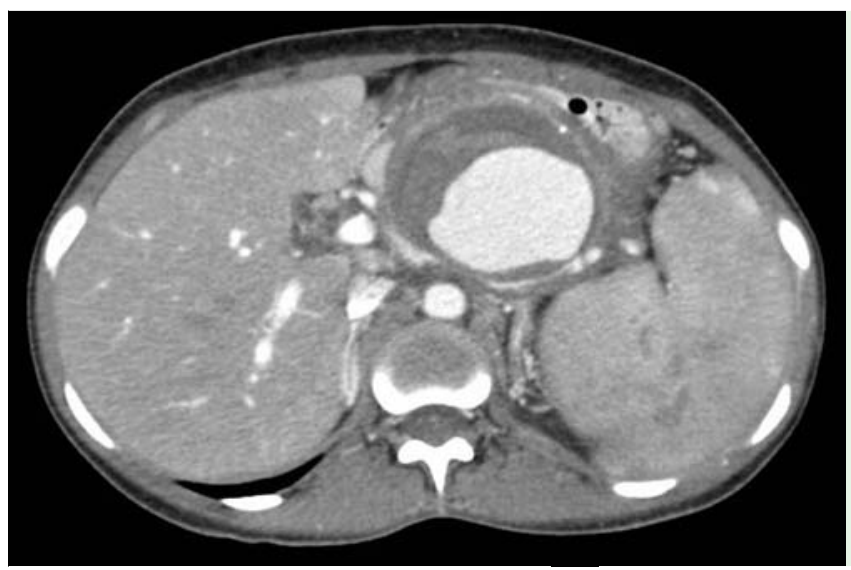

Fig. 1 Computed tomography angiography showing a large splenic artery pseudoaneurysm.

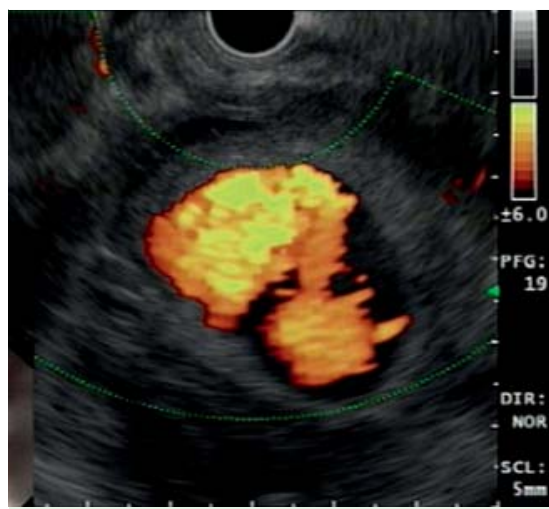

Fig. 2 Endoscopic ultrasound with color Doppler, showing splenic artery pseudoaneurysm with blood flow.

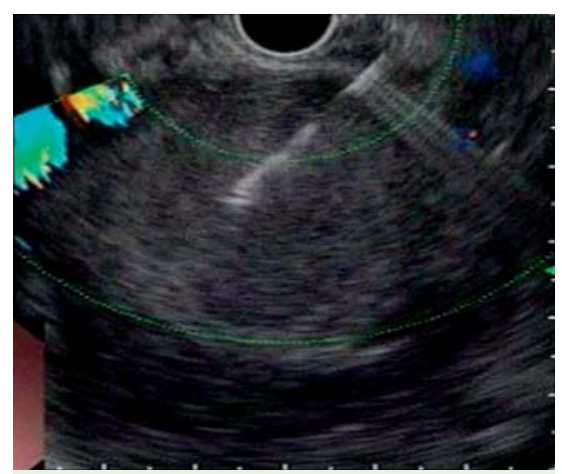

Fig. 3 Endoscopic ultrasound showing a thrombosed pseudoaneurysm following thrombin injection.

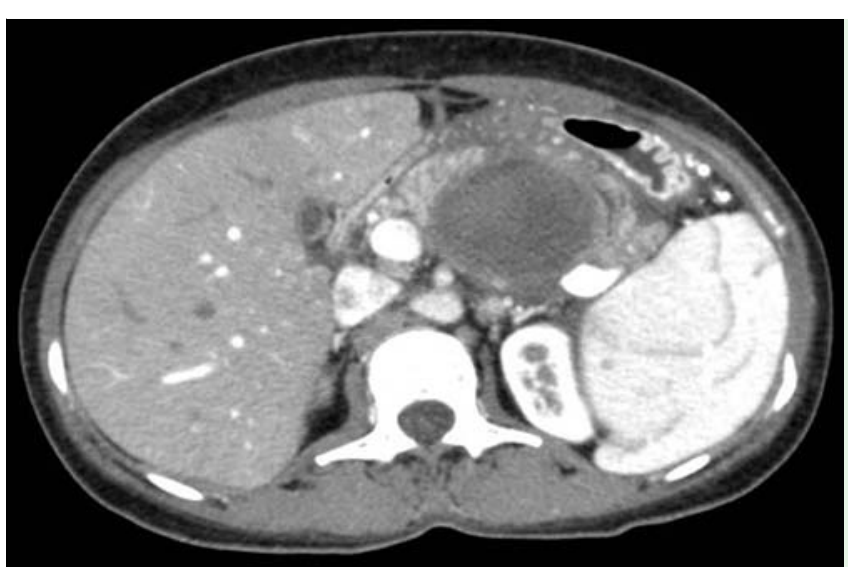

year but had worsened during the past 15 days. The general and systemic examination was normal. Hemogram and liver function tests were normal. Serum amylase was $450 \mathrm{U} / \mathrm{L}$ (normal value $\leq 150 \mathrm{U} / \mathrm{L}$ ). Abdominal ultrasound revealed multiple calcifications in a dilated pancreatic duct, peripancreatic inflammation, and a splenic artery pseudoaneurysm. Computed tomography (CT) angiography confirmed acute and chronic pancreatitis with a partially thrombosed splenic artery pseudoaneurysm of size $6.5 \times 6.0 \times 6.8 \mathrm{~cm}$ (๑ Fig. 1).

The patient was treated with analgesics for pain. She could not afford to undergo coil angioembolization of the pseudoaneurysm as this procedure is expensive. Endoscopic ultrasound (EUS)-guided thrombin injection was therefore planned as this procedure is significantly cheaper, and was possible due to the close proximity of the lesion to the gastric wall and the narrow neck of the pseudoaneurysm.

The pseudoaneurysm was located by using a linear echoendoscope (GF-UCT180; Olympus Medical Systems, Tokyo, Japan) ( $\bullet$ Fig. 2). A standard 22-gauge needle (Cook Endoscopy, Winston-Salem, North Carolina, USA) was used to puncture the pseudoaneurysm and inject $2 \mathrm{~mL}$ of thrombin. Color Doppler confirmed the complete obliteration of the pseudoaneurysm ( Fig.3). The patient was asymptomatic after the procedure and was discharged after 48 hours. CT angiography at 6 months showed no recurrence of the pseudoaneurysm ( $\bullet$ Fig.4).

There are a few other reports of EUS-guided thrombin injection for visceral pseudoaneurysm [4,5]. Further studies are needed to establish its use as a first-line treatment for visceral pseudoaneurysms.

\section{Video 1}

Endoscopic ultrasound sequence of large splenic artery pseudoaneurysm. Injection of $2 \mathrm{~mL}$ of thrombin resulted in thrombosis of the pseudoaneurysm. Color Doppler was used to show blood blow and confirm the thrombosed pseudoaneurysm.

Endoscopy_UCTN_Code_TTT_1AS_2AC

\section{Competing interests: None}

Visceral pseudoaneurysm is an uncommon but serious complication of acute or chronic pancreatitis. It affects the splenic artery in $40 \%$ of cases [1]. Traditional treatment includes surgical excision or ligation and endovascular techniques [2, 3]. This article describes the first reported case of a large splenic artery pseudoaneurysm that was managed by endoscopic ultrasound (EUS)-guided thrombin injection in a patient with tropical chronic pancreatitis.

A 25-year-old woman presented with epigastric pain, which had persisted for 1 


\section{Praveer Rai ${ }^{1}$, Shantam Mohan', Malay Sharma ${ }^{2}$}

${ }^{1}$ Department of Gastroenterology, Sanjay Gandhi Postgraduate Institute of Medical Sciences, Lucknow, India

${ }^{2}$ Department of Gastroenterology, Jaswant Rai Superspeciality Hospital, Meerut, India

\section{References}

1 Mallick IH, Winslet MC. Vascular complications of pancreatitis. J Pancreas (Online) 2004; 5: 328-337

2 Balachandra S, Siriwardena A. Systematic appraisal of the management of the major vascular complications of pancreatitis. Am J Surg 2005; 190: 489-495

3 Tulsyan N, Kashyap VS, Greenberg RK et al. The endovascular management of visceral artery aneurysms and pseudoaneurysms. J Vasc Surg 2007; 45: 276-283

4 Roach H, Roberts SA, Salter $R$ et al. Endoscopic ultrasound-guided thrombin injection for the treatment of pancreatic pseudoaneurysms. Endoscopy 2005; 37: 876878

5 Robinson M, Richards D, Carr N. Treatment of a splenic artery pseudoaneurysm by endoscopic ultrasound-guided thrombin injection. Cardiovasc Intervent Radiol 2007; 30: 515-517
Bibliography

Dol http://dx.doi.org/

10.1055/s-0034-1377357

Endoscopy 2014; 46: E355-E356

(c) Georg Thieme Verlag KG

Stuttgart · New York

ISSN 0013-726X

\section{Corresponding author}

\section{Praveer Rai, MD, DM}

Department of Gastroenterology

Sanjay Gandhi Postgraduate Institute of Medical Sciences

Lucknow 226014

India

Fax: +91-522-2494598

praveer_rai@yahoo.com 